\title{
The effect of gender differences on the use of valproic acid for migraine prophylaxis
}

\author{
(D) Nermin Tepe \\ Balıkesir University, Faculty of Medicine, Department of Neurology, Balıkesir, Turkey
}

Cite this article as: Tepe N. The effect of gender differences on the use of valproic acid for migraine prophylaxis. J Health Sci Med 2021; 4(4): 408-411.

\begin{abstract}
Aim: The efficacy of the drugs used for migraine prophylaxis remains unclear. At our headache polyclinic, when we observed the difference in prophylactic response especially in valproic acid (VA) treatment between genders, the response of male was found to be better than that of females so we wanted to document this difference by collecting data retrospectively

Material and Method: Forty-six chronic migraine without aura patients with VA who were enrolled in our headache outpatient clinic between 2015-2018 were included in the study (29 male, 17 female). In these patients, after VA the frequency of montly attacks between sexes, changes in visual analogue scale (VAS) scores, single VA prophylaxis and the addition of a second prophylaxis drug were evaluated.

Results: None of the men needed an additional second drug while 11 of 17 women needed the addition of a second drug. There was no gender difference in monthly attack frequency before treatment, whereas monthly attack frequency after treatment with VA was found to be significantly lower in men (before treatment; female $20.9 \pm 11.3$ male $17.7 \pm 11.5$, p $>0.05$ ) (after treatment; male $1.2 \pm 5.5$ female $12.0 \pm 12.2, \mathrm{p}<0.05)$. There was no difference between sexes in the pre-treatment VAS scores, but the VAS score was significantly lower in men after treatment with VA (before treatment; female $7.8 \pm 0.8$ male $8.1 \pm 1.2, p>0.05)($ after treatment; female $4.4 \pm 2.8$ male $2.3 \pm 2.8, \mathrm{p}<0.05) .75 .9 \%$ of men had no post-treatment migraine attacks, while this rate was $5.9 \%$ in women $(\mathrm{p}<0.05)$. The dose range of VA treatment wasn't found to be different between genders.

Conclusion: Better response of men with migraine to valproic acid treatment is important in clinical practice, and the reason for this situation may be clarified with further studies.
\end{abstract}

Keywords: Migraine, valproic acid, visual analogue scale

\section{INTRODUCTION}

Migraine is a multifactorial neurovascular syndrome characterized by episodes of moderate to severe, unilateral, pulsating headaches with nausea and/or vomiting, worsened by light and sound, pain associated with triggering factors in genetically susceptible individuals (1). Diagnostic criteria established by the International Headache Society (IHS) are used in the diagnosis of migraine (2). The most frequent migraine episodes are encountered in the second and third decades, with the highest incidence of episodes occurring between the ages of 35 and 45 years. The course of the disease is usually with attacks, but migraine is chronic in about $4 \%$ of the patients. Migraine treatment is given acutely and/ or prophylactically. Recurring migraine that significantly interferes with the patient's daily routine despite acute treatment. For example, two or more migraine attacks/ month impairing function for $\geq 3$ days or infrequent migraine attacks that produce profound disability during each attack/ failure of, contraindication to, or intolerable side effects to acute medications/ acute medication overuse or the risk of developing acute medication overuse prophylactic treatment should be given (3). Effective prophylactic treatment should reduce at least $50 \%$ of the monthly episode frequency within three months (3). Among beta blockers, especially propranolol, valproate and topiramate from antiepileptics, flunarizine from calcium channel blockers, and amitriptyline from tricyclic antidepressants are used for migraine prophylaxis. Topiramate and valproate are antiepileptics approved by the FDA for migraine treatment. Valproic acid can be given in a single dose 500-1800 mg/ day. Valproate has effects like providing neuronal 
hyperpolarization by enhancing potassium transport and increasing postsynaptic GABA response. Various studies using valproate have reported reductions in migraine attack frequency by $43 \%$ to $48 \%(4,5)$. The studies have used valproate at doses ranging from 500 to $1500 \mathrm{mg}$ daily for migraine prophylaxis have demonstrated that valproate is significantly more effective than placebo $(6,7)$. Prophylactic response varies among patients who take valproate at the same dosage. In our practice, we wanted to document this difference by collecting data recorded at our headache polyclinic retrospectively due to the obvious difference between genders, especially in valproate treatment.

\section{MATERIAL AND METHOD}

This study is a retrospective study and the study was approved by the Clinical Researches Ethics Committee of Balıkesir University, Faculty of Medicine (Date: 20.02.2019, Decision No: 2019/34). All procedures were carried out in accordance with the ethical rules and the principles of the Declaration of Helsinki.

Forty-six chronic migraine without aura patients who received valproic acid between 2015 and 2018 were enrolled in our headache outpatient clinic (29 men, 17 women). International Headache Institute (IHS) diagnostic criteria were used to diagnose migraine without aura in our outpatient clinic (2). The VAS pain scale, age, sex, prophylactic drugs used, presence of comorbid systemic diseases, frequency of monthly migraine headaches and time of migraine attack onset were recorded. Patients with systemic diseases, psychiatric comorbidities, $>65$ years of age were not included. After valproic acid, monthly attack frequency and severity, VAS score, drug dose results in the 1st, 3rd and 6th months were also recorded. We usually start valproic acid treatment with $250 \mathrm{mg}$ dosage in a single dose and give information to patients about $250 \mathrm{mg}$ increments weekly to reduce the adverse effects, and advise on how to decide to increase the dosage by weekly increments. Some patients have responded to a $500 \mathrm{mg}$ controlled release valproic acid tablet dosage, while some others required a dose of $750 \mathrm{mg}$ or $1000 \mathrm{mg}$.

\section{Statistical Analysis}

The data from this research was electronically transferred to the SPSS 20.0 statistics program, and data control and analysis were performed. Descriptive statistics (frequency, percentage distribution, mean, median) were used as statistical analysis. ManWhitney-U was used in the comparison of quantitative data as inferential statistics method test, and chi-square test was used to compare qualitative data. $\mathrm{P}<0.05$ was considered to be a statistically significant value.

\section{RESULTS}

The mean age of female patients included in the study was $54.7 \pm 10.2$ years, while the mean age of male migraine patients was $46.3 \pm 11.5$ years. $75.9 \%$ of men had no migraine attacks post-treatment, while this rate was $5.9 \%$ in women $(p=0.001)$. There was no gender difference in the monthly attack frequency before treatment, whereas the monthly attack frequency after treatment with valproic acid was found to be significantly lower in men (before treatment; female $20.9 \pm 11.3$ male $17.7 \pm 11.5, \mathrm{p}=0.38$ ) (after treatment; male $1.2 \pm 5.5$ female $12.0 \pm 12.2, \mathrm{p}=0.001$ ) (Figure 1). There was no difference between genders in the pre-treatment VAS scores, but the VAS score was significantly lower in men after treatment with valproic acid (before treatment; female $7.8 \pm 0.8$ male $8.1 \pm 1.2, \mathrm{p}=0.31$ ) (after treatment; female $4.4 \pm 2.8$ male $2.3 \pm 2.8, \mathrm{p}=0.02$ ) (Figure 2). The median dose of valproic acid was found to be $1000 \mathrm{mg}$ in women and $750 \mathrm{mg}$ in men, there was no statistically significant difference. None of the men needed the addition of a second drug while 11 of 17 women needed the addition of a second prophylaxis drug $(\mathrm{p}=0.001)$ (Figure 3$)$.

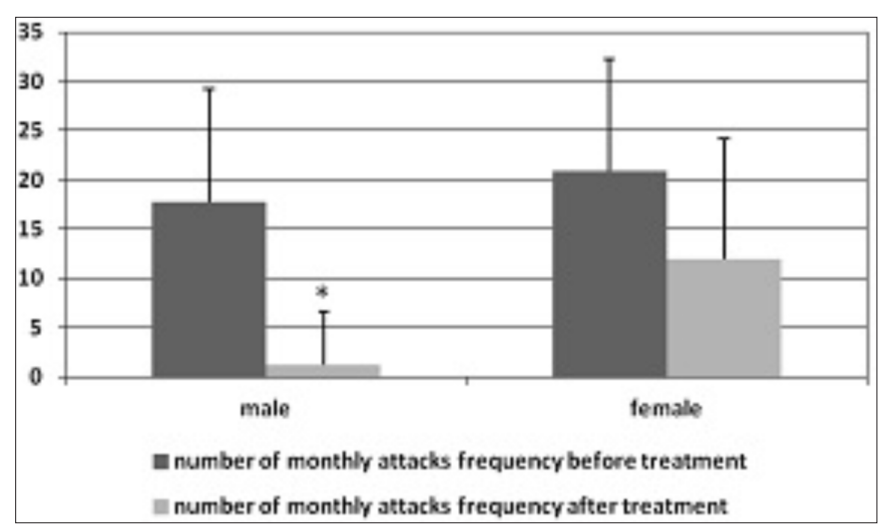

Figure 1. Monthly attack frequency (before- after valproic acid); The monthly attack frequency was found to be statistically significant in males compared to females. $75,9 \%$ of men had no migraine attack post-treatment, while this rate was $5,9 \%$ in women $(\mathrm{p}<0,05)$.

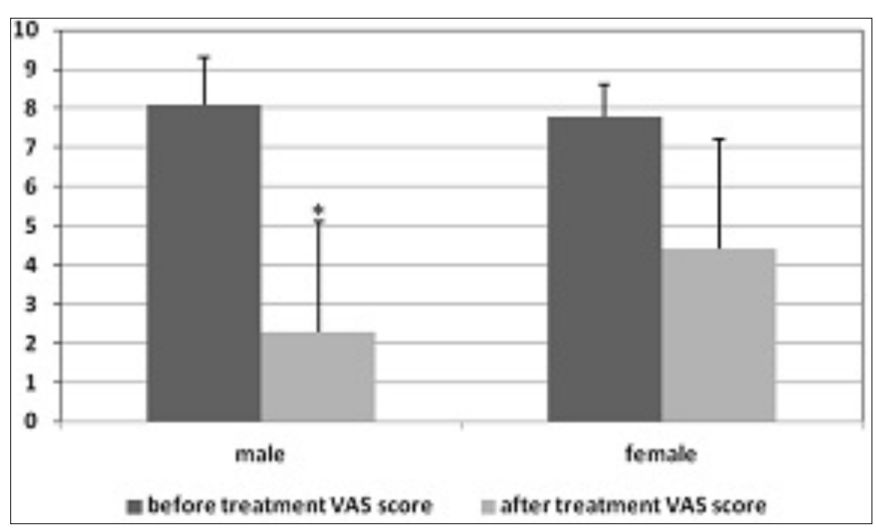

Figure 2. VAS score was found to be statistically significant in males compared to females. (before treatment; female 7,8 $\pm 0,8$ male $8,1 \pm 1,2$ ) (after treatment; female $4,4 \pm 2,8$ male $2,3 \pm 2,8, \mathrm{p}<0,05$ ) $(\mathrm{mean} \pm \mathrm{SD})$. VAS score (before- after valproic acid) 


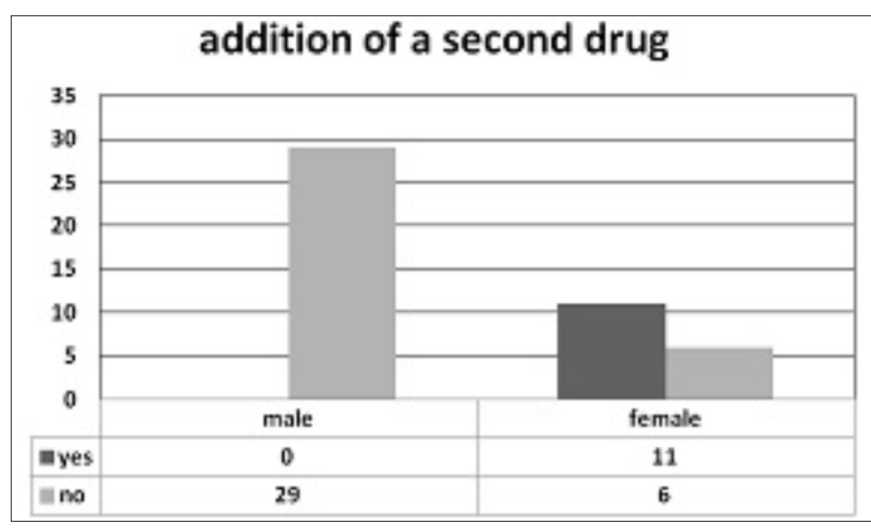

Figure 3. None of the men needed the addition of a second drug while 11 of 17 women needed the addition of a second prophylaxis drug $(\mathrm{p}<0,05)$

\section{DISCUSSION}

The response to prophylactic therapy varies among migraine patients. Various studies have shown that polymorphic changes in drug metabolism genes, which display differences among individuals, are responsible for $20-40 \%$ of the individuals' responses to the drugs and $50 \%$ of their side effects (8). The association between antiepileptic drugs and genetic polymorphisms has been evaluated in many studies (9-11). In the current study, no statistical significance was detected between the response to other drugs used in the prophylactic treatment of migraine and the related gene polymorphisms (except for topiramate). The results suggest that migraine patients who receive topiramate prophylactic treatment should be assessed for the presence of the MDR1 C3435T polymorphism. However, there was no significant relationship between the treatment response to topiramate and either the CYP2D6 or CYP2C19 polymorphism, and there were no significant correlation between the treatment responses to amitriptyline, propranolol, and valproate and the MDR1, CYP2D6 and CYP2C19 gene polymorphisms (12). In one study, no significant or nominal association with genetic load was found for any of the prophylactic treatments, either in combination or separately (13). There is currently no solid evidence that pharmacogenomics should be used in migraine prophylaxis. Variability of the dose-response mainly depends on three factors: 1- factors associated with the disease mechanism, which are unknown for prophylactic migraine drugs; 2- pharmacodynamics, also unknown for such drugs; 3- pharmacokinetic factors. Metoprolol, propranolol, and amitriptyline have high pharmacokinetic variability, whereas valproate, topiramate, atenolol, naproxen, and candesartan have low pharmacokinetic variability; however, factors determining this variability are poorly understood (14). Another study shows that age and gender significantly influence valproate serum concentrations but several publications have reported no effects of age on total valproate serum concentrations
$(15,16)$. In our study, the frequency of attacks was independent of age in male patients who responded to valproate therapy. Ibarra et al. have shown increased reabsorbed fraction and bioavailability of valproate doses in females compared to males, hence indicating gender differences in hepatobiliary output, which emerge as higher bioavailability of valproate in females than males (17). These studies cannot help to explain why valproate is more effective in male patients.

An examination of recent studies revealed no evaluations on the response to valproate prophylaxis for migraine headache in both genders. After observing that valproate decreases the frequency and severity of migraine headache, especially in men, the results of the present retrospective study showed a decrease in attack frequency and VAS score, which was statistically significant. In addition, the effect that valproic acid alone is sufficient for prophylaxis in men and the need for adding a second drug in women are high and statistically significant, supports the gender differences in the response of valproic acid. Moreover, while valproate prophylaxis alone was sufficient in men, the rate of addition of the second drug was high and statistically significant in women and these data support the differences in valproic acid responses between genders. It still remains unclear why valproate has a different effect on migraine depending on gender. As a continuation of this study, we will search new polymorphisms for valproate with the drug blood level combination for the difference in migraine prophylaxis responses in both sexes.

The present study had several potential limitations. Firstly, this study was designed as a retrospective study. Secondly, since our study was retrospective, we could not look at the valproic acid blood levels of our patients. Thirdly our number patients were small.

\section{CONCLUSION}

We wanted to share our clinical observation with this study. Monotherapy is primarily the basis for migraine prophylaxis, and the fact that valproic acid is more effective in men than women will contribute to clinical practice.

\section{ETHICAL DECLARATIONS}

Ethics Committee Approval: The study was approved by the Clinical Researches Ethics Committee of Balıkesir University, Faculty of Medicine (Date: 20.02.2019, Decision No: 2019/34).

Informed Consent: Because the study was designed retrospectively, no written informed consent form was obtained from patients. 
Referee Evaluation Process: Externally peer-reviewed.

Conflict of Interest Statement: The authors have no conflicts of interest to declare.

Financial Disclosure: The authors declared that this study had received no financial support.

Author Contributions: All of the authors declare that they have all participated in the design, execution, and analysis of the paper and that they have approved the final version.

Acknowledgment: For critical review support thanks to Dr. Gülseren Büyükşerbetçi.

\section{REFERENCES}

1. Bolay $H$, Moskowitz MA. The neurobiology of migraine and transformation of headache therapy. In 'Neuroscience, Molecular Medicine and the Therapeutic Transformation of Neurology' 2004; 107- 23.

2. Headache Classification Committee of the International Headache Society (IHS). The International Classification of Headache Disorders (ICHD), 3rd edition. Cephalalgia 2018; 38:1-211.

3. Parikh SK, Silberstein SD. Preventive Treatment for Episodic Migraine. Neurol Clin 2019; 37: 753-70.

4. Freitag FG, Collins SD, Carlson HA, et al. A randomized trial of divalproex sodium extended-release tablets in migraine prophylaxis. For the Depakote ER Migraine Study Group. Neurology 2003; 58: 1652-9.

5. Klapper JA. Divalproex sodium in migraine prophylaxis: a dosecontrolled study. Cephalalgia 1997;17: 103-8.

6. Linde M, Mulleners WM, Chronicle EP, McCrory DC. Valproate (valproic acid or sodium valproate or a combination of the two) for the prophylaxis of episodic migraine in adults. Cochrane Database Syst Rev 2013; CD010611.

7. Tatyana S, Jae-Young C, Rema R, et alreventive pharmacologic treatments for episodic migraine in adults. J Gen Intern Med 2013; 28: 1225-37.

8. Bertilson L. Pharmacogenomics: drugs and the pharmaceutical science, in Historical Aspects of Pharmacogenetics 2001; 1-9.

9. Saygi S, Alehan F, Atac FB, Erol I, Verdi H, Erdem R. Multidrug resistance 1 (MDR1) 3435C/T genotyping in childhood drugresistant epilepsy. Brain Dev 2014; 36: 137-42.

10. Ozgon GO, Bebek N, Gul G, Cine N. Association of MDR1 (C3435T) polymorphism and resistance to carbamazepine in epileptic patients from Turkey. Eur Neurol 2008; 59: 67-70.

11.Li M, Tan J, Yang X, et al. The ABCB1-C3435T polymorphism likely acts as a risk factor for resistance to antiepileptic drugs. Epilepsy Res 2014; 108: 1052-67.

12. Atasayar G, Eryilmaz IE, Karli N, et al. Association of MDR1, CYP2D6, and CYP2C19 gene polymorphisms with prophylactic migraine treatment response. J Neurol Sci 2016; 366:149-54.

13. Christensen AF, Esserlind AL, Werge T, Hreinn S, Stefa'nsson K, Olesen J. The influence of genetic constitution on migraine drug responses. Cephalalgia 2016; 36: 624-39.

14.Tfelt-Hansen P, Nybye A, Pavbro A, Tfelt-Hansen J. Pharmacokinetic variability of drugs used for prophylactic treatment of migraine. CNS Drug 2017; 31: 389- 403.

15.Smith RL, Haslemo T, Refsum H, Molden E. Impact of age, gender and CYP2C9/2C19 genotypes on dose-adjusted steadystate serum concentrations of valproic acid-a large-scale study based on naturalistic therapeutic drug monitoring data. Eur J Clin Pharmacol 2016; 72: 1099-104.
16. Perucca E, Grimaldi R, Gatti G, Pirracchio S, Crema F, Frigo GM. Pharmacokinetics of valproic acid in the elderly. Br J Clin Pharmacol 1984; 17: 665-69.

17.Ibarra M, Vazquez M, Fagiolino P, Derendorf H. Sex-related differences on valproic acid pharmacokinetics after oral single dose. J Pharmacokinet Pharmacodyn 2013; 40: 479-86. 